\title{
Referral Patterns in Oral Medicine: A Retrospective Analysis of an Oral Medicine University Center in Southern Italy
}

\author{
Noemi Coppola ${ }^{1}$, Stefania Baldares ${ }^{1}$, Andrea Blasi ${ }^{1, *}$, Rosaria Bucci ${ }^{2} \mathbb{D}$, Gianrico Spagnuolo ${ }^{1,3}{ }^{\mathbb{D}}$, \\ Michele Davide Mignogna ${ }^{1}$ and Stefania Leuci ${ }^{1}$ (D)
}

1 Oral Medicine Unit, Department of Neuroscience, Reproductive and Odontostomatological Sciences, University of Naples Federico II, 80131 Naples, Italy; noemi.coppola@unina.it (N.C.); dott.baldares@tiscali.it (S.B.); gspagnuo@unina.it (G.S.); mignogna@unina.it (M.D.M.); stefania.leuci@unina.it (S.L.)

2 Department of Neuroscience, Reproductive and Odontostomatological Sciences, Section of Orthodontics and Temporomandibular Disorders, University of Naples Federico II, 80131 Naples, Italy; rosaria.bucci@unina.it

3 Institute of Dentistry, I. M. Sechenov First Moscow State Medical University, 119435 Moscow, Russia

* Correspondence: andreablasi79@gmail.com; Tel.: +39-389-342-9887

check for updates

Citation: Coppola, N.; Baldares, S.; Blasi, A.; Bucci, R.; Spagnuolo, G.; Mignogna, M.D.; Leuci, S. Referral Patterns in Oral Medicine: A Retrospective Analysis of an Oral Medicine University Center in Southern Italy. Int. J. Environ. Res. Public Health 2021, 18, 12161. https:/ / doi.org/10.3390/ijerph182212161

Academic Editor: Paul B. Tchounwou

Received: 13 October 2021

Accepted: 11 November 2021

Published: 19 November 2021

Publisher's Note: MDPI stays neutral with regard to jurisdictional claims in published maps and institutional affiliations.

Copyright: (c) 2021 by the authors. Licensee MDPI, Basel, Switzerland. This article is an open access article distributed under the terms and conditions of the Creative Commons Attribution (CC BY) license (https:/ / creativecommons.org/licenses/by/ $4.0 /)$.

\begin{abstract}
Referral of a patient from one healthcare provider to another is an important part of the medical practice. The aim of this study was to analyze the referral process to the Oral Medicine Unit in a university-based tertiary center in Southern Italy. A chart review of new referrals to the Oral Medicine Unit during a 24-month period was conducted. The following data were recorded: demographic characteristics, medical history, number of physicians seen prior to Oral Medicine assessment, referral source, diagnostic procedures ordered by referrals, reason for referral, site of lesion/condition, final diagnosis. Then, the rates of correct identification for health-care professionals and the appropriateness of the reference diagnosis based on the disease were calculated with descriptive statistic indicators. There were 583 new first consultations. A total of $62.9 \%$ of patients were referred by general dental practitioners, $27.4 \%$ by physicians, and $9.7 \%$ did not have a referral. The most common diseases for referral were immune-mediated diseases (39.6\%) and oro-facial pain disorders $(25.2 \%)$. Only $28.5 \%$ of patients had a correct provisional diagnosis. The results of this study show the need to implement curricula in the field of oral medicine among dentistry and medical students, and to support the continuing education among healthcare providers to reduce diagnostic delay for oral diseases.
\end{abstract}

Keywords: oral medicine; oral diseases; referral; quality of referral; consultation; primary healthcare; dentistry

\section{Introduction}

Referral is an integral part and a critical component of the current medical practice, defined as the taking over care from one healthcare provider to another [1]. The referring physician sends the patient to the specialist physician for evaluation and management of a specific clinical problem [2]. If practised efficiently, it can contribute to high standards of care by improving patient outcomes and decreasing costs through optimal use of medical services. High-quality referral and consultation processes require good communication between healthcare providers: this may lead to reduced delays in the diagnostic care pathway, high-quality continuity of care and improved patient's satisfaction [3]. The interaction between general medical practitioner (GMP) and specialist physician is based on the recommendation letters where there is the patient's clinical history, the description of symptoms and a provisional diagnostic hypothesis [4]. In medicine, the analysis of referral patterns is a relevant and current issue as it is closely connected to the quality of healthcare and its costs [5]. To provide quality healthcare by ensuring that the patient receives proper diagnosis and treatment in a timely manner, the referral must have requirements of necessity, 
adequacy, timeliness and must be well communicated in the letters of recommendation [6]. Furthermore, the quality of the referral process assumes even greater importance in the current historical moment as we are witnessing, on the one hand, an increase in the prevalence of patients that are in chronic maintenance for multiple pathologies (oncological, immunological, neurological, etc.) and, on the other hand, the scarcity of resources to satisfy the demand for specialized care [7]. Referral patterns, therefore, have significant implications in the field of health policies (workforce planning, hospital, and academic funding policy) [8]. Access to oral medicine (OM) is generally based on referrals. OM is defined as "The discipline of dentistry concerned with the oral healthcare of medically complex patients including the diagnosis and management of medical conditions that affect the oral and maxillofacial region" [9]. Scopes of OM are not unique worldwide; in fact, there is no international consensus on the definition and field of action of OM [10]. For example, in Europe, OM is recognised as a specialty only in Croatia, Israel and the United Kingdom. In some European countries, it is an integrated course of study in other dental specialties; in other countries, however, such as in Italy, Spain and Sweden, training in OM is a separate course from other recognized dental disciplines [10]. There is therefore no single training course for OM, which unfortunately is completely lacking in some countries. Despite the great variability depending on the country to which reference is made, OM specialists $(\mathrm{OMs})$ care for patients with a wide range of conditions, including mucosal diseases, oral manifestations of systemic diseases, oral drug-related complications, salivary gland disorders, orofacial pain and temporomandibular conditions [11]. OM inherently has strong relationships with various medical specialities, particularly dermatology, immunology and infectious diseases, pathology, imaging, neurology, oncology, ear-nose-throat specialist (ENT), paediatrics, psychiatry, psychology and rheumatology [12]. Thus, OM is placed at the interface between medicine and dentistry and should ideally serve as a model for interdisciplinary collaboration. According to "Oral Medicine referral guidelines in UK", the referral letter should include the urgency of the referral, a detailed history of the patient's complaint, details about prior investigations or treatment performed and a provisional diagnosis, using a red flag for suspicious lesions [13]. Moreover, using high-quality photos may improve the referral process [13]. Retrospective studies on OM referral patterns have been conducted in various countries (Ireland, United States, Australia) [14-16]. However, at present, similar data concerning Italy are not available in the literature. Our study aims to describe the different aspects of the referral process to the Oral Medicine Unit (OM-U) in a university-based tertiary referral center located in Southern Italy. In particular, we analyzed source, reason, pertinence and accuracy of the referral diagnosis in the recommendation letters, with the aim of providing a detailed and current picture of services provided in our university and of their use by the dental and medical community in general.

\section{Materials and Methods}

This descriptive study was conducted at the OM-U of the University of Naples Federico II, a specialty referral clinic (Naples, Campania, Italy). This clinic is one of the two referral centers for OM in this region and serves a population of about 5,600,000 (2021). The majority of patients comes from local area; however, some patients from other regions come to this center. We reviewed retrospectively all the new patient' charts, referred from 1 March 2019 to 31 December 2020. The second year of patient enrollment was marked by the COVID pandemic; therefore, the main enrollment period can be considered until February 2020. Patients who were referred for suspicious oral medicine-related illnesses were included. Patients for whom it was not possible to collect data in detail and for whom a final diagnosis was not indicated were excluded. Each patient underwent a complete clinical interview and examination performed by an OMs.

\subsection{Data Collection}

Data were extracted and recorded from an electronic database by a single researcher. The following variables were identified: age, gender, smoker status (light smokers: $<5$ cigarettes 
daily; moderate smokers: $5-10$ cigarettes daily; heavy smokers: 15 or more cigarettes daily), alcohol consumption (light drinker: one unit of alcohol daily; moderate drinker: two units/daily; heavy drinker: three or more units/daily), medical history, current drugs, number of physicians seen prior to OM assessment, diagnostic procedures ordered by referrals, referral source, reason for referral, site of lesion/condition, time elapsed between referral and visit to OM-U, final diagnosis by the OMs in according with the International Statistical Classification of Diseases and Related Health Problems (International Classification of Diseases, 11th edition [ICD-11] codes) [17]. Then, the final diagnosis was analyzed by a second researcher in order to categorize into general diagnostic categories proposed by Villa et al.: (1) immune-mediated mucosal conditions, (2) orofacial pain (OFP) disorders, (3) benign tumors or neoplasms, (4) dysplasia and cancerous conditions, (5) reactive keratosis, (6) salivary gland disorders, (7) infections, (8) osteonecrosis of the jaw and (9) other mucosal/gingival and bone conditions [15].

\subsection{Statistical Analysis}

For continuous variables (age, n. of physicians seen prior to OM assessment and time elapsed between referral and visit to OM-U) mean and standard deviation we calculated, whereas non continuous variables (gender, smoking status, alcohol consumption, referral source, diagnostic procedures, reasons for referral, site of the lesion, accuracy of initial diagnosis and category of final diagnosis) were reported as frequencies and percentages. A statistical software (IBM SPSS Statistics v.25, IBM Inc., Armonk, NY, USA) was used for calculation.

\section{Results}

\subsection{Patient Demographic Characteristics}

The demographic characteristics of patients are show in Table 1. During the study period, a total of 583 patients were seen for the first OM consultation. The average age was $56.6 \pm 16.2 ; 218(37.4 \%)$ were males and $365(62.6 \%)$ females. Almost all patients were of Caucasian ethnicity with only 2 from different ethnic groups.

Table 1. The demographic characteristic of patients.

\begin{tabular}{llll}
\hline & & $\mathbf{N}$ & $(\%)$ \\
\hline All patients & 583 & \\
Age, mean years \pm SD & $56.6 \pm 16.2$ & \\
Gender & & \\
Male & 218 & 37.4 \\
Female & 365 & 62.6 \\
Risk factor & & \\
Smoking status & & \\
Never & 308 & 52.8 \\
$<5$ & 120 & 20.6 \\
$5-15$ & 113 & 19.4 \\
$>15$ & 42 & 7.2 \\
Alcohol use & & \\
Never & 215 & 36.9 \\
Light drinker & 219 & 37.6 \\
Moderate drinker & 127 & 21.8 \\
Heavy drinker & 22 & 3.8 \\
\hline
\end{tabular}

The number of non-smokers were $308(52.8 \%)$, whereas the number of smokers was $275(47.2 \%)$. Regarding alcohol consumption, 215 (36.9\%) patients refrained completely from alcohol consumption, and $368(63.1 \%)$ reported consuming alcohol.

\subsection{Referral Source}

A total of 1163 consultations were performed prior to referral to our university clinic: 439 patients $(75.4 \%)$ have consulted one or two doctors, $51(8.8 \%)$ consulted three and $72(12.4 \%)$ consulted more than three. Figure 1 shows in detail the sources of the referral. 
Of the total sample, 366 patients $(62.9 \%)$ were referred by general dental practitioners (GDPs), $160(27.4 \%)$ by physicians and $57(9.7 \%)$ did not have a referral. From physicians, 48 (8.2\% of total referrals) were primary care physicians (PCPs), 26 (4.5\%) maxillofacial surgeons, 25 (4.3\%) dermatologists and $16(2.7 \%)$ ENT.

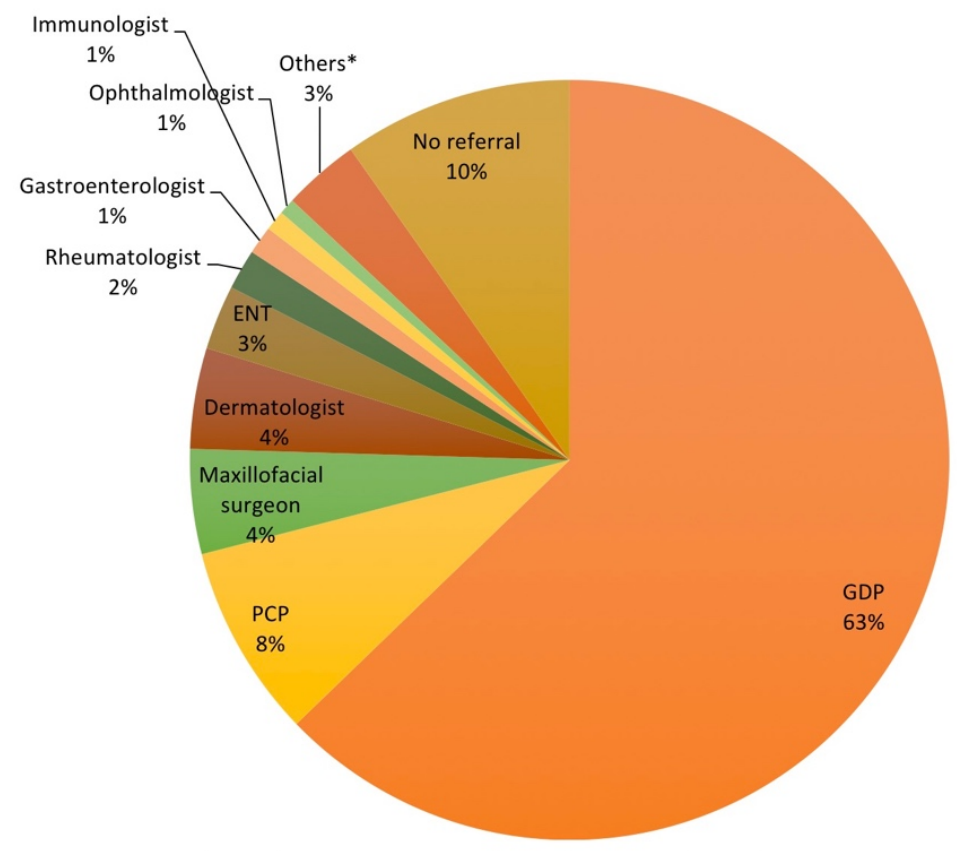

Figure 1. Source of referral. * Others: Oncologist, Pathologist, Plastic surgeon, Neuropsychiatric, Pediatrician, Breast specialist, Allergist, Cardiologist, Hematologist, Gynecologist, Internist, Neurologist. ENT: ear-nose-throat specialist; PCP: primary care physician; GDP: general dental practitioner.

\subsection{Diagnostic Procedures Ordered by Referral}

Before the OM visit, 229 patients (39.3\%) underwent diagnostic procedures, the most common of which were: routine blood test (13.7\%), biopsy (9.6\%) and panoramic radiograph (orthopantomogram-OPG) $(9.1 \%)$. The other diagnostic tests ordered by referrals are presented in Table 2. Some patients have performed more than one diagnostic test. On the basis of the final diagnosis the appropriateness of the required tests was $41.9 \%(96 / 229)$. The condition for which more incongruous diagnostic tools were ordered was burning mouth syndrome (BMS).

Table 2. Diagnostic tests ordered by referring providers.

\begin{tabular}{lll}
\hline & Diagnostic Test & N \\
\hline Blood examination & 80 & \\
Biopsy & 56 & \\
Orthopantomogram & 53 & \\
Lingual swab & 42 \\
Computed tomography maxillo-facial & 26 \\
Gastroscopy & 10 \\
Maxillo-facial MRI & 8 \\
Brain MRI & 6 & \\
General urine test & 2 \\
Others & 25 \\
\hline
\end{tabular}

\subsection{Reasons for Referral}

Table 3 shows in details the reason for referral in according to final diagnosis either clinically or histopathologically by OMs. The most common reason for referral was immune-mediated mucosal conditions diagnosed in 231 patients (39.6\%) followed by OFP 
disorders $(147 / 583 ; 25.2 \%)$ and dysplasia and cancerous conditions $(81 / 583 ; 13.9 \%)$. From immune-mediated mucosal disease, the most frequent diagnosis was oral lichen planus (OLP) $(164 / 583 ; 28.1 \%$ of total diagnosis), whereas, from the OFP group, BMS (111/583; $19 \%$ ) was the main diagnosis, and from dysplasia and cancerous conditions the most common diagnosis was leukoplakia $(51 / 583 ; 8.8 \%)$.

Table 3. Reasons for referral.

\begin{tabular}{|c|c|c|}
\hline Disease & $\mathbf{N}$ & $(\%)$ \\
\hline Immune-mediated mucosal conditions & 231 & 39.6 \\
\hline Oral lichen planus & 164 & 28.1 \\
\hline Orofacial granulomatosis & 29 & 5 \\
\hline Recurrent aphthous & 19 & 3.3 \\
\hline Geographic tongue & 18 & 3.1 \\
\hline Pemphigus disease & 13 & 2.2 \\
\hline Plasma cell mucositis & 6 & 1 \\
\hline Pemphigoid disease & 4 & 0.7 \\
\hline Erythema multiforme & 3 & 0.5 \\
\hline Behcet's disease & 1 & 0.2 \\
\hline Orofacial pain disorders & 147 & 25.2 \\
\hline Burning mouth syndrome & 111 & 19 \\
\hline Persistent idiopathic facial pain & 30 & 5.1 \\
\hline Trigeminal neuralgia & 4 & 0.7 \\
\hline Myofascial pain & 1 & 0.2 \\
\hline Globus pharyngeus & 1 & 0.2 \\
\hline Benign tumors or neoplasms & 32 & 5.5 \\
\hline Fibroma & 20 & 3.4 \\
\hline HPV-related lesions & 8 & 1.4 \\
\hline Peripheral giant cell granuloma & 2 & 0.3 \\
\hline Lipoma & 1 & 0.2 \\
\hline Varix & 1 & 0.2 \\
\hline Dysplasia and cancerous conditions & 81 & 13.9 \\
\hline Leukoplakia & 51 & 8.7 \\
\hline Oral carcinoma & 27 & 4.6 \\
\hline Erythroplakia & 3 & 0.5 \\
\hline Reactive keratosis & 12 & 2 \\
\hline Salivary gland disorders & 11 & 1.9 \\
\hline Sjogren's syndrome & 6 & 1 \\
\hline Mucocele & 3 & 0.5 \\
\hline Sialadenitis & 5 & 0.9 \\
\hline Infections (Oral candidiasis) & 15 & 2.6 \\
\hline Osteonecrosis of the jaws & 13 & 2.2 \\
\hline Other mucosal and gingival lesions & 22 & 3.8 \\
\hline Others * & 16 & 2.7 \\
\hline
\end{tabular}

* Other conditions not included in the previous categories (dental pathology, normal variants, no disease).

\subsection{Accuracy of Referrals}

Only approximately half of the patients was referred with a provisional diagnosis ( 308 of $583 ; 52.9 \%$ ) and, of these, $63.9 \%$ (166 of 308) of the initial diagnoses were confirmed (Figure 2).

The rates of correct identification of the three most frequent conditions, OLP, BMS and leukoplakia, were the following. On a total of 164 cases of OLP, only 97 had a provisional diagnosis and, of these, only $56(57.7 \%)$ were determined to be correct. A total of 51 out of 111 BMS patients were referred with initial diagnosis and $21(41.2 \%)$ were appropriate. From 51 patients with a diagnosis of leukoplakia, 30 had a diagnosis from the referring provider and only $2(6.7 \%)$ were consistent with final diagnosis. Osteonecrosis of the jaws, pemphigus and pemphigoid, aphtosis were the conditions with the highest degree of diagnostic accuracy $(91.7 \%, 66.7 \%, 66.7 \%$ and $58.3 \%$, respectively). The rates of correct identification for health-care professionals (HCP) were the following: GDPs $(68 / 195$; 
$34.9 \%)$, PCPs (14/26; 53.8\%), maxillofacial surgeon (7/16; 43.7\%), ENT (7/13; 53.6\%). The diagnostic accuracy of the other physicians is shown in Figure 3.

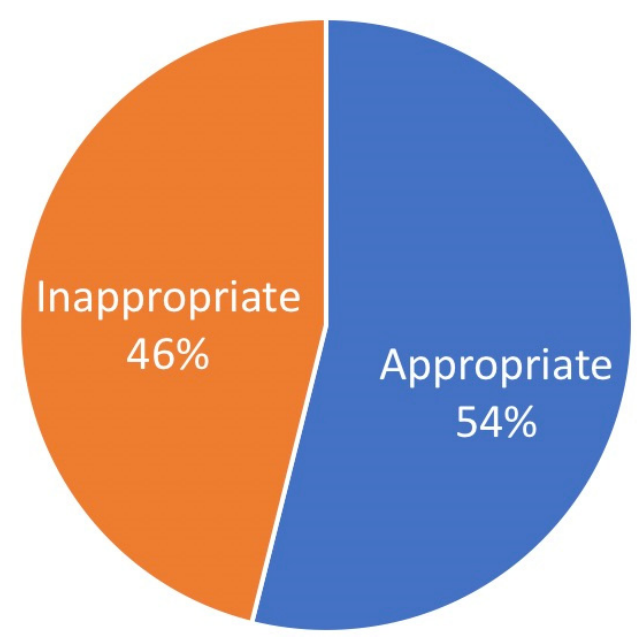

Figure 2. Appropriateness of referral diagnosis.

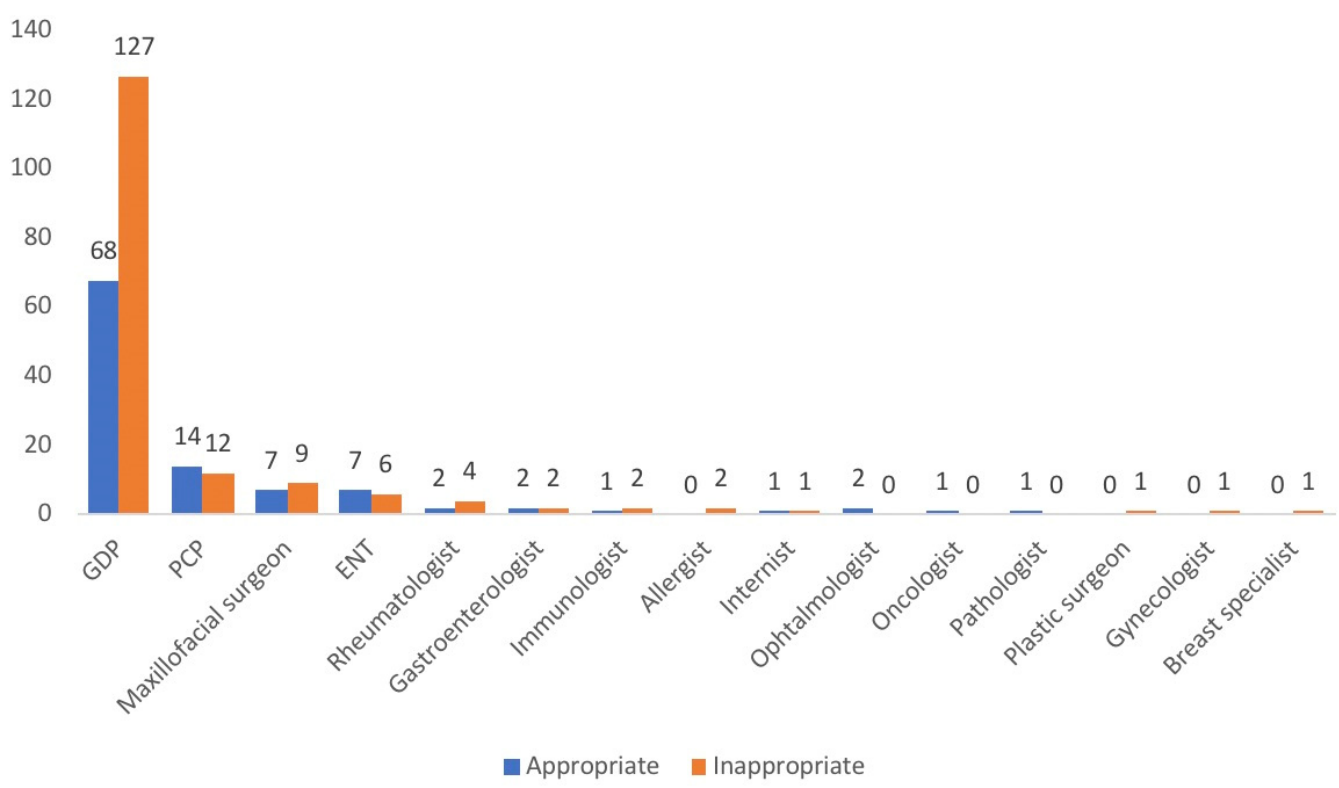

Figure 3. Appropriateness of the reference diagnosis based on the specialty of the referring provider.

\section{Discussion}

This single-center study examines the referral pattern related to the OM-U of a University-based hospital in Naples (Italy). In our cohort and as previously reported, the majority of patients were in the fifth decade of life with a female prevalence $[15,18]$. These data suggest that, to date, OM services are aimed at an adult population and the aging of the general population will increase the demand for assistance in this field [19]. Therefore, with increasing requests, the referral process to the OMs must be appropriate and timely to improve access to healthcare, coordination of care and not waste public health resources. About $75 \%$ of patients were visited by at least one doctor before being referred; on average, the number of medical consultations seen is two. The most common sources of referral were GDPs, PCPs, maxillofacial surgeons and dermatologists in line with data from Friesen et al., and Farah et al. [16,18]. Only $27 \%$ came from physicians; this percentage is in line with the studies conducted in Australia and in Canada, but in disagreement with Villa et al., where $2 / 3$ of patients were referred by physicians $[15,16,18]$. A recent systematic review on knowledge, attitude and practice among healthcare providers in oral cancer 
awareness shows that most of dentists usually referred patients to specialists, instead a minority of medical doctors preferred to refer to specialists, which was rarely an OMs [20]. On the one hand, this can be explained with the different levels of cooperation between dentists and physicians in the healthcare system of various countries, as is also supported by Farah et al. [16]. On the other hand, the low percentage of patients referred by medical doctors could indicate that there is no information among the medical community about the services offered by OM. Moreover, is widely demonstrated that most medical providers consider inadequate the education regarding oral health issues, and they reported that they rarely examine the oral cavity [21,22]. Consequently, the reduced number of referrals by physicians could also be attributed to the non-recognition of oral pathologies due to the lack of oral inspection. Therefore, there are two important needs to improve the management of OM patients. First, it is imperative to raise awareness of the support that OM can provide in the healthcare system. Second, it is also important to encourage continuing training courses in oral medicine for physicians. Although each patient consulted two doctors on average before $\mathrm{OM}$ consultation, only half of the patients had a provisional diagnosis and, on a total sample, only $28.5 \%$ of patients had an initial diagnosis which was then clinically or histologically confirmed by OMs. GDPs, PCPs and ENT had a higher degree of diagnostic appropriateness among HCPs without a statistically significant difference between dentists and physicians, according to Friesen et al., and Sardella et al. [18,23]. The low rate of diagnostic accuracy makes early diagnosis difficult and highlights that dentists and physicians are not confident with oral diagnosis, and are unable to perform the proper management of clinical patients. This confirms that oral medicine is a specialty which is still finding its place in absence of an international consensus. Whereas in the past years OM have been placed between medicine and dentistry, to date, it is defined as a branch of dentistry even in the absence of shared legislation in the various countries $[10,24]$. The great diversity both from an educational and clinical point of view worldwide means that there are few healthcare professionals able to satisfy the request for assistance from patients. In particular, to regard the diagnostic procedures, in our study emerged that before the referral to OMs the referring provider ordered or performed diagnostic tool only in $40 \%$ of patients. In Italy, diagnostic procedures are provided by the national health system and the choice of resorting to private facilities to carry out medical consultations and assessments depends solely on the will of the patient. Routine blood test followed by biopsy and OPG was the most diagnostic procedure for the assessment of the disease. Compared with the data in the literature, there is a higher percentage of blood examinations and a lower percentage of biopsies performed by other medical professionals, but the biopsy appropriateness rate was higher than in other studies (75\%). In fact, in the study by Villa et al., $17.1 \%$ of patients had undergone biopsy before the visit with OMs, but more than 1/3 of the biopsies performed were deemed inadequate [15]. Additionally, in our cohort, many diagnostic tests were not appropriate for the patient's condition, mostly for BMS patients, such as lingual swab, biopsy, and esophago-gastro-duodenoscopy, according to the International Classification of Orofacial Pain [25]. These data are in line with a recent study by Freilich et al., who reported that many BMS patients, despite requiring medical attention, have undergone unnecessary tests and have not been diagnosed or misdiagnosed [26]. Among oral diseases, certainly, BMS is still a challenge for clinicians and for patients $[27,28]$, but any diagnosis in the field of OM is frequently complex. In our study, patients were referred for very heterogeneous diseases, OLP and BMS were the most frequent of them. OFP disorders were the second reasons for referral in line with the increase in the prevalence of chronic pain worldwide, the most common reasons why people seek medical attention [29-31]. Although the most frequent pathologies were OLP, BMS and leukolpakia, the most easily diagnosed were osteonecrosis of the jaw bones and autoimmune bullous diseases. These data were in contrast to what emerges from the study by Sardella et al. [23]. In the case of osteonecrosis, the high diagnostic accuracy may be due to the exposure of necrotic bone, pathognomonic feature of this pathology and easy sign to detect. On the other hand, the presence of a highly sensitive and specific test, such as the ELISA test for the detection of 
anti-desmoglein-1, anti-desmoglein-3, anti-BP180 and anti-BP230 antibodies, may have contributed to the diagnostic precision towards autoimmune bullous diseases. Despite the high percentage of patients with oral cancer risk factors in our cohort, there were relatively few diagnoses of cancerous lesions. Finally, a small percentage of referrals $(2.6 \%)$ was for dental pathology and paraphysiologic conditions (no evident mucosal pathology, torus mandibularis, tongue ptosis).

As stated above, a referral letter should also include photographs. In our cohort no case was provided with photographic documentation. Several authors suggested that clinical images can be useful for an initial consultation and prioritize [32-35].

Our study shows that patients with oral diseases are often seen by multiple clinicians before being evaluated by an OMs and may be subjected to unnecessary and often inappropriate diagnostic tests, procedures and therapies. This results in diagnostic delay and delay in the referral to OM-U could worsen the prognosis for many conditions or at least affect the patients' oral health-related Quality of Life. The high percentage of patients without diagnosis and provisional misdiagnosis indicates the absence of expert figures in $\mathrm{OM}$ among health professionals. Furthermore, the high percentage of referrals from dentists indicates that they are the health professionals who are responsible for intercepting the first signs and symptoms of oral diseases. Therefore, to achieve the goal of reducing diagnostic delay and improving healthcare for patients with oral diseases, it is necessary to implement the pre- and post-graduate training education of the dental classes. Only by investing in a broader cultural path can a turning point be reached in the management of patients requesting assistance for $\mathrm{OM}$ issues. The study has, however, different limitations. First, the relatively small sample size of the group, which was influenced by the COVID pandemic that limited the access of patients to hospital facilities. Second, the method of retrospective chart review that does not allow to analyse other factors in the referral. Third, this is a single-center study; therefore, the results are not generalizable on a large-scale population.

\section{Conclusions}

This study analyzes the referral pattern to OM in Italy, highlighting the growing demand for assistance in this field related to the high incidence of oral diseases and the crucial role that OM services play. The results of this study demonstrate a low grade of correct diagnosis by referral provider with the consequent need to implement curricula among dentistry and medical students. Finally, it is imperative that OM becomes even more of a reference for the medical community as well, in order to provide a complete medical assistance to patients.

Author Contributions: Conceptualization, S.L. and N.C.; methodology, N.C.; software, A.B.; validation, N.C., S.L. and M.D.M.; formal analysis, A.B.; investigation, S.B. and G.S.; resources, S.B. and R.B.; data curation, S.B. and N.C.; writing-original draft preparation, N.C., S.L.; writing-review and editing, M.D.M.; visualization, A.B.; supervision, S.L.; project administration, R.B. and G.S.; funding acquisition, G.S. All authors have read and agreed to the published version of the manuscript.

Funding: This research received no external funding.

Institutional Review Board Statement: The study was conducted according to the guidelines of the Declaration of Helsinki, and approved by the Institutional Review Board of University of Naples Federico II (Approval Code 437/2020).

Informed Consent Statement: Informed consent was obtained from all subjects involved in the study.

Data Availability Statement: The data presented in this study are available on request from the corresponding author.

Conflicts of Interest: The authors declare no conflict of interest. 


\section{References}

1. Hensher, M.; Price, M.; Adomakoh, S. Referral hospitals. In Disease Control Priorities in Developing Countries, 2nd ed.; Chapter 66; Jamison, D.T., Breman, J.G., Measham, A.R., Alleyne, G., Claeson, M., Evans, D.B., Jha, P., Mills, A., Musgrove, P., Eds.; Oxford University Press and The World Bank: New York, NY, USA, 2006.

2. Mehrotra, A.; Forrest, C.B.; Lin, C.Y. Dropping the baton: Specialty referrals in the United States. Milbank Q. 2011, 89, 39-68. [CrossRef]

3. Guevara, J.P.; Hsu, D.; Forrest, C.B. Performance measures of the specialty referral process: A systematic review of the literature. BMC Heal. Serv. Res. 2011, 11, 168. [CrossRef] [PubMed]

4. Dickie, J.A.; Ellwood, D.A.; Robertson, M. What's in a referral letter: Does the detail matter? Australas. J. Ultrasound Med. 2011, 14, 11-14. [CrossRef] [PubMed]

5. Sampson, R.; Cooper, J.; Barbour, R.; Polson, R.; Wilson, P. Patients' perspectives on the medical primary-secondary care interface: Systematic review and synthesis of qualitative research. BMJ Open 2015, 5, e008708. [CrossRef]

6. Tobin-Schnittger, P.; O’Doherty, J.; O'Connor, R.; O'Regan, A. Improving quality of referral letters from primary to secondary care: A literature review and discussion paper. Prim. Health Care Res. Dev. 2017, 19, 211-222. [CrossRef]

7. Tzartzas, K.; Oberhauser, P.-N.; Marion-Veyron, R.; Bourquin, C.; Senn, N.; Stiefel, F. General practitioners referring patients to specialists in tertiary healthcare: A qualitative study. BMC Fam. Pract. 2019, 20, 1-9. [CrossRef] [PubMed]

8. Moore, K. Social Determinants of Health: Addressing Barriers Through Screening, Referrals, and Care Coordination. North Carol. Med. J. 2019, 80, 306-311. [CrossRef] [PubMed]

9. American Academy of Oral Medicine. Available online: http://www.aaom.com/ (accessed on 25 September 2021).

10. Bez, C.; Sklavounou, A.; Carrozzo, M. Oral medicine in Europe: Past, present and future. Br. Dent. J. 2017, 223, 726-728. [CrossRef] [PubMed]

11. Scully, C.; Miller, C.S.; Urizar, J.-M.A.; Alajbeg, I.; Almeida, O.P.; Bagan, J.V.; Birek, C.; Chen, Q.; Farah, C.; Figueirido, J.P.; et al. Oral medicine (stomatology) across the globe: Birth, growth, and future. Oral Surg. Oral Med. Oral Pathol. Oral Radiol. 2015, 121, 149-157. [CrossRef] [PubMed]

12. Spielman, A.I. The future of oral medicine. Oral Dis. 2018, 24, 285-288. [CrossRef]

13. Available online: https://www.england.nhs.uk/south/wp-content/uploads/sites/6/2019/02/mcn-oral-medicine-referralguidelines-for-gdps-jan-2019-v1.pdf (accessed on 5 November 2021).

14. Riordain, R.; O'Sullivan, K.; McCreary, C. Retrospective evaluation of the referral pattern to an oral medicine unit in Ireland. Community Dent. Health 2011, 28, 107-110. [CrossRef]

15. Villa, A.; Stock, S.; Aboalela, A.; Lerman, M.A.; Woo, S.-B.; Sonis, S.T.; Treister, N.S. Oral Medicine referrals at a hospital-based practice in the United States. Oral Surg. Oral Med. Oral Pathol. Oral Radiol. 2015, 119, 423-429. [CrossRef] [PubMed]

16. Farah, C.S.; Simanovic, B.; Savage, N.W. Scope of practice, referral patterns and lesion occurrence of an oral medicine service in Australia. Oral Dis. 2008, 14, 367-375. [CrossRef]

17. ICD-11. International Classification of Diseases 11th Revision. Available online: https://icd.who.int/en. (accessed on 17 November 2021).

18. Friesen, R.; McGaw, T.; Peters, E.; Lai, H. A retrospective analysis of referral patterns to a university oral medicine clinic. Oral Surg. Oral Med. Oral Pathol. Oral Radiol. 2019, 128, 381-385. [CrossRef] [PubMed]

19. Patterson, J.; Willis, A. Oral Medicine Considerations for Older Patients. Prim. Dent. J. 2020, 9, 45-53. [CrossRef]

20. Coppola, N.; Mignogna, M.; Rivieccio, I.; Blasi, A.; Bizzoca, M.; Sorrentino, R.; Muzio, L.L.; Spagnuolo, G.; Leuci, S. Current Knowledge, Attitudes, and Practice among Health Care Providers in OSCC Awareness: Systematic Review and Meta-Analysis. Int. J. Environ. Res. Public Health 2021, 18, 4506. [CrossRef]

21. Vernon, L.T.; Teng, K.A.; Kaelber, D.C.; Heintschel, G.P.; Nelson, S. Time to integrate oral health screening into medicine? A survey of primary care providers of older adults and an evidence-based rationale for integration. Gerodontology 2021. [CrossRef]

22. Guan, G.; Lau, J.; Yew, V.; Jiayun, U.; Qu, W.; Lam, J.; Mei, L. Referrals by general dental practitioners and medical practitioners to oral medicine specialists in New Zealand: A study to develop protocol guidelines. Oral Surg. Oral Med. Oral Pathol. Oral Radiol. 2020, 130, 43-51.e5. [CrossRef] [PubMed]

23. Sardella, A.; Demarosi, F.; Lodi, G.; Canegallo, L.; Rimondini, L.; Carrassi, A. Accuracy of Referrals to a Specialist Oral Medicine Unit by General Medical and Dental Practitioners and the Educational Implications. J. Dent. Educ. 2007, 71, 487-491. [CrossRef] [PubMed]

24. Schmidt-Westhausen, A.M.; Bornstein, M.M. Orale Medizin: Interdisziplinäre Zusammenarbeit zwischen Medizin und Zahnmedizin [Oral medicine: A specialty placed between medicine and dentistry]. Bundesgesundheitsblatt Gesundheitsforschung Gesundheitsschutz 2011, 54, 1061-1065. [CrossRef]

25. Benoliel, R.; May, A.; Svensson, P. International Classification of Orofacial Pain, 1st edition (ICOP). Cephalalgia 2020, 40, $129-221$.

26. Freilich, J.E.; Kuten-Shorrer, M.; Treister, N.S.; Woo, S.-B.; Villa, A. Burning mouth syndrome: A diagnostic challenge. Oral Surg. Oral Med. Oral Pathol. Oral Radiol. 2019, 129, 120-124. [CrossRef] [PubMed]

27. Klasser, G.D.; Epstein, J.B.; Villines, D.; Utsman, R. Burning mouth syndrome: A challenge for dental practitioners and patients. Gen. Dent. 2011, 59, 210-220. [PubMed]

28. Mignogna, M.D.; Fedele, S.; Russo, L.L.; Leuci, S.; Muzio, L.L. The diagnosis of burning mouth syndrome represents a challenge for clinicians. J. Orofac. Pain 2005, 19, 168-173. 
29. Vos, T.; Abajobir, A.A.; Abate, K.H.; Abbafati, C.; Abbas, K.M.; Abd-Allah, F.; Criqui, M.H. GBD 2016 Disease and Injury Incidence and Prevalence Collaborators. Global, regional, and national incidence, prevalence, and years lived with disability for 328 diseases and injuries for 195 countries, 1990-2016: A systematic analysis for the Global Burden of Disease Study 2016. Lancet 2017, 390, 1211-1259.

30. Colloca, L.; Ludman, T.; Bouhassira, D.; Baron, R.; Dickenson, A.H.; Yarnitsky, D.; Freeman, R.; Truini, A.; Attal, N.; Finnerup, N.B.; et al. Neuropathic pain. Nature reviews. Dis. Primers 2017, 3, 17002. [CrossRef] [PubMed]

31. Crocetto, F.; Coppola, N.; Barone, B.; Leuci, S.; Imbimbo, C.; Mignogna, M.D. The association between burning mouth syndrome and urologic chronic pelvic pain syndrome: A case-control study. J. Oral Pathol. Med. 2020, 49, 829-834. [CrossRef]

32. Aslam, A.; Hamburger, J. Does the use of photography help to prioritise patients when referring to the oral medicine department? Br. Dent. J. 2010, 208, E16. [CrossRef]

33. Petruzzi, M.; De Benedittis, M. WhatsApp: A telemedicine platform for facilitating remote oral medicine consultation and improving clinical examinations. Oral Surg. Oral Med. Oral Pathol. Oral Radiol. 2016, 121, 248-254. [CrossRef]

34. Pentapati, K.C.; Smriti, K.; Gadicherla, S. WhatsApp: A telemedicine platform for facilitating remote oral medicine consultation and improving clinical examinations-a commentary. Oral Surg. Oral Med. Oral Pathol. Oral Radiol. 2016, 121, 573. [CrossRef]

35. Carrard, V.C.; Martins, M.A.T.; Molina-Bastos, C.G.; Gonçalves, M.R. WhatsApp: A telemedicine platform for facilitating remote oral medicine consultation and improving clinical examinations-some considerations. Oral Surg. Oral Med. Oral Pathol. Oral Radiol. 2017, 123, 408. [CrossRef] [PubMed] 